\title{
Numerical 3D Simulation of a Longitudinal Ventilation System: Memorial Tunnel Case
}

\author{
Galdo Vega, M.; Argüelles Díaz, K.M.; Fernández Oro, J.M.; \\ Ballesteros Tajadura, R.; Santolaria Morros, C. \\ Universidad de Oviedo, Área de Mecánica de Fluidos. \\ Campus de Viesques, 33271, Gijón (Asturias), Spain. \\ arguelleskatia@,uniovi.es
}

\begin{abstract}
In this work, a numerical 3D simulation of a longitudinal ventilation system (LVS) is developed to analyze the fire behaviour inside a road tunnel. The numerical modelling reproduces the Memorial Tunnel, a two-lane, $853 \mathrm{~m}$ long road tunnel, used for experimental purposes. On this tunnel, 98 full-scale fire ventilation tests with different ventilation systems were conducted, constituting the first significant experimental approach to analyze fire incidents inside road tunnels. A total number of 24 reversible jets fans were installed in groups of three, nearly equally spaced over the length of the tunnel, and cantilevered from the ceiling of the tunnel. The validation of a numerical model is developed in the present paper. For that purpose, the behaviour of the smoke generated during a fire incident inside a road tunnel is predicted and compared with previous experimental data collected in the Memorial Tunnel Project. The smoke evolution and the performance of the LVS is simulated with a commercial code, FLUENT, which allows 3D unsteady simulations of the Navier-Stokes equations for multispecies mixtures of gases. A sufficient mesh density was introduced for the spatial discretization in order to obtain accurate results in a reasonable CPU time. Hence, typical ratios between total number of cells and the overall tunnel length were employed in the modelling. As a result, good agreement was achieved in all the tested cases, defining an accurate methodology to predict the performance of a LVS in case of fire inside a tunnel.
\end{abstract}

\section{INTRODUCTION}

Fires in road tunnels are extremely hazardous for drivers and rescue teams. Recent disasters, like crashes in the Mont Blanc tunnel (France, 1999) or San Gottardo (Italy, 2001), have shown the need for better integral actions in the case of fire incidents. In particular, the minimum delay time required for starting the jet fans, or the evolution of the smoke patterns inside the tunnel are critical issues when rescue plans are designed. Since major interest is placed on developing systems that minimize both material and personal damages, the design of ventilation systems must be focused on the life protection, while the evacuation of the tunnel is completed. Therefore, these ventilation systems must incorporate an integral operating manual in which the airflow necessary to control the smoke or the direction and schedule of fresh-air supply should be clearly specified. Unfortunately, there is not just one simple procedure to estimate these operating conditions because of the inherent complexity of the problem: a non-linear convection of multispecies mixture of gases inside one particular tunnel geometry, function of heat and energy sources (fire characteristics) and weather conditions (variable boundary conditions).

Three different methodologies can be cited as basic tools to study the smoke propagation in case of fire: pseudo-thermal scale models, full scale tests and numerical models. In the first case, significant contributions using the pseudo-thermal method can be found in the bibliography ([1], [2], [3]), though their results are difficult to extrapolate to different operating conditions and are quite limited to low temperature applications. The second method (full scale tests) is really expensive and only two of them have been conducted recently: the EUREKA Project [4] and the Memorial Tunnel Fire Ventilation Test Program [5]. Because they require of large experimental facilities, they are difficult to operate and maintain in order to obtain good results. The last method (numerical modelling), under development, consists of customizing CFD codes to analyze the fire evolution when the ventilation system operates inside meshed tunnel geometries (see for example references[6], [7], [8] \& [9]). However, in order to be completely reliable, these codes must be calibrated and verified through comparison with experimental data. 
The present paper establishes the modelling framework to be applied in case of simulation of LVSs [10] through a direct comparison with the results of the Memorial Tunnel Project.

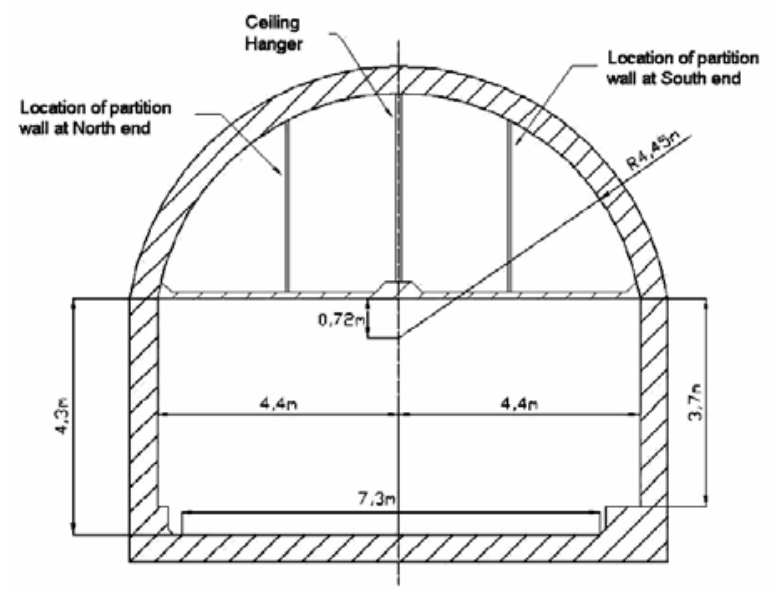

Figure 1. Memorial Tunnel cross section.

The Memorial Tunnel Fire Ventilation Test Program (MTFVTP) was a comprehensive, full-scale test program, conducted to provide definitive data to support ventilation system design. The Memorial Tunnel is a two-lane, $853 \mathrm{~m}$ long road tunnel, built in 1953 near Charleston, West Virginia (USA.), with a 3.2 percent upgrade from the South to the North tunnel portal. The cross-section of the tunnel, excluding the ceiling, is approximately $60.39 \mathrm{~m}^{2}$ (figure 1). Originally, the tunnel ventilation system was fully transverse, consisting of a supply fan chamber at the South portal and an exhaust fan chamber at the North portal. For the experiments, it was modified and instrumented to allow operation and evaluation of different ventilation systems such as transverse (both full and partial) and longitudinal ventilation with jet fans, and even natural ventilation. The effectiveness of all these ventilation systems to manage the smoke evolution over time and the temperature distributions obtained inside the tunnel were tested in case of different heat powers: 10, 20, 50 and $100 \mathrm{MW}$.

The program of the Memorial Tunnel Project consisted in 98 full-scale ventilation tests, 15 of them performed to analyze the response of the LVS. A total number of 24 jet fans were installed in groups of three, nearly equally spaced over the total length of the tunnel, and cantilevered from the ceiling of the tunnel. In this particular case, the tunnel fake ceiling was removed (it was used only for transverse ventilation). Every fan was driven by a $75 \mathrm{HP}$ AC motor, delivering $155000 \mathrm{~m}^{3} / \mathrm{h}$ of air at a discharge velocity of $34.2 \mathrm{~m} / \mathrm{s}$. The first 12 tests were operated using only 15 jet fans. Nine additional units were included in the facility when conducting the three remaining tests. The fire location inside the tunnel, showed in figure 2 , was maintained in the same location of all the 15 cases under LVS conditions.

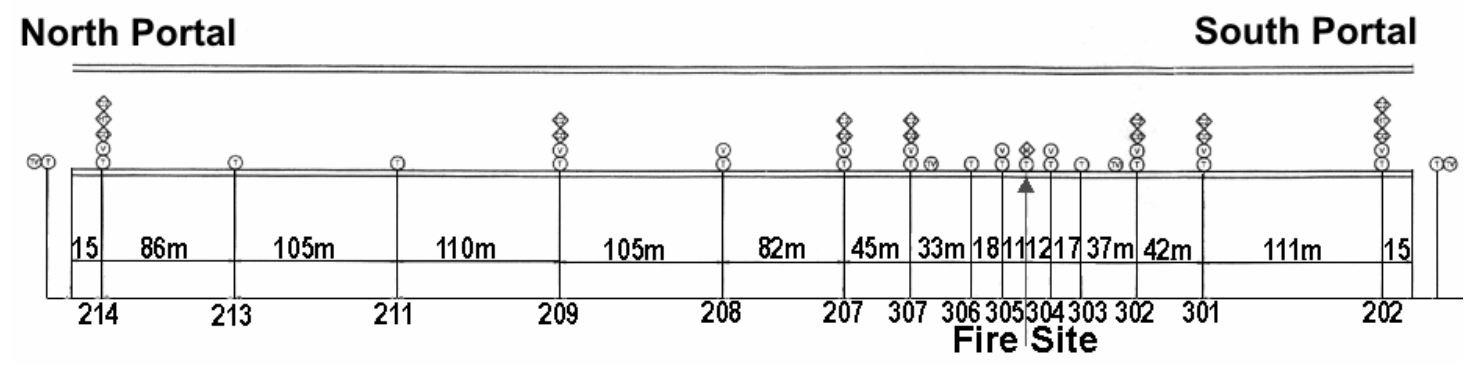

Figure 2. Fire location inside the tunnel.

In addition, the Memorial Tunnel was equipped with multiple sensors in order to collect data of different variables, such as fire temperature, velocity, carbon dioxide or carbon monoxide concentration. These all-type sensors were arranged in instrumentations trees, referred to as "loops" in the Project, and distributed in representative sections of the tunnel (figure 2). All the measured values were classified and recorded in a database, resulting in a valuable collection of data sets, including point-in-time graphics, time history graphs and a brief summary of the test conditions. The test summary is a list with the test number, the nominal size of the fire input, the sequence of the events in the test and additional important information. Point-in-time graphics show instantaneous snapshots of the variables distribution and time history graphs trace the evolution of the different variables during the test. Comparison of the events sequence (delay and response time, operating conditions and so on) with the evolution of all the variables measured by the "loops", indicates the ability of the LVS to manage the smoke dispersion, depending on the fire size and the fans response time.

The validation of the numerical modelling has been carried out using three representative tests among all the 15 LVS cases:

1) Test No. 606A (figure 3.a): Only two jet fans, placed far away from the fire situation and with a response time of 5 minutes, are operated with a 10 MW heat rate.

2) Test No. 612B (figure 3.b): The heat rate is increased to $50 \mathrm{MW}$, with five jet fans operating in a row (the top central one). The response time was maintained in 5 minutes.

3) Test No.611 (figure 3.c): The response time of the system is reduced to 2 minutes, but a different 
layout of the operative fans is selected. The fans are now concentrated around the $50 \mathrm{MW}$ heat rate with a $2+3$ configuration.

Graphics and results derived from these three tests provided useful data to validate a $3 \mathrm{D}$ numerical model reproducing similar conditions of the Memorial Tunnel. The complete three-dimensional definition of the tunnel geometry was developed using a commercial code, FLUENT, which includes a species transport model to simulate the inclusion of carbon dioxide particles released from the heat sources. An unsteady scheme was also introduced to trace the evolution of the carbon monoxide concentration inside the tunnel, and standard $\mathrm{k}-\varepsilon$ model was employed for the turbulence modelling.
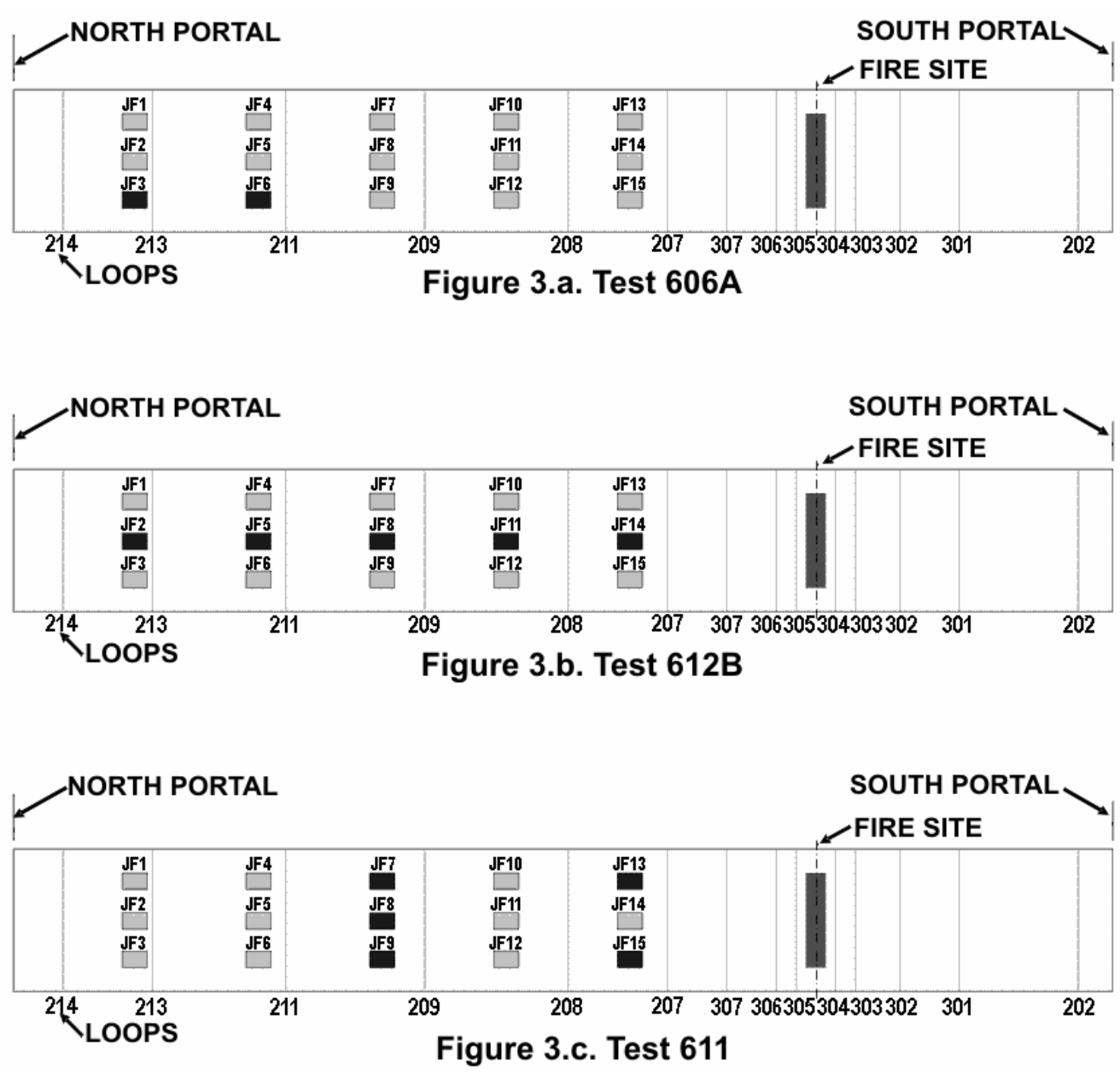

Figure 3. Layout of operative fans in the tests.

\section{METHODOLOGY}

A CFD code is employed in the present work to reproduce the Memorial Tunnel tests, solving the differential equations for the conservation of mass, momentum and energy and closing the equation set with standard turbulence modelling. Additionally, it is necessary to solve a convection-diffusion equation for all the species involved in the mixture of gases. Hence, defining a local mass fraction of each species, $\alpha_{i}$, a conservation equation following the general form has to be implemented in the numerical modelling. An equation of this form will be solved for $N-1$ species where $N$ is the total number of fluid phase chemical species present in the system. Since the mass fraction of the species must sum to unity, the $N_{t h}$ mass fraction is determined as one minus the sum of the $N-1$ solved mass fractions. In this case, two species where introduced in the model, air and $\mathrm{CO}_{2}$.

The calculations were performed using a commercial software package, FLUENT v.6.0, which uses the finite volume method to solve the threedimensional Navier-Stokes equations on an unstructured grid. The turbulence was simulated with the standard k- $\varepsilon$ model. For such calculations, wall functions, based on the logarithmic law, have been used. Gravitational body forces were included in the momentum equations, defining buoyancy terms function of the temperature variations according to the Boussinesq approach. The scheme for the timedependent term was second order, implicit. Additionally, second order, upwind discretization was used for convective terms and a central difference scheme was used for diffusive terms. 

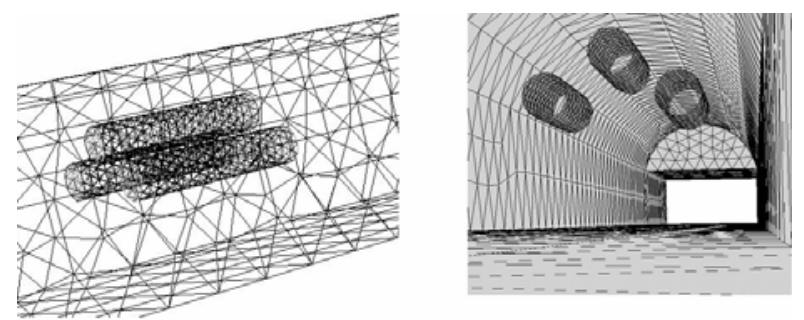

Jet Fans Location

Figure 4. Detailed mesh for the jet fans region.
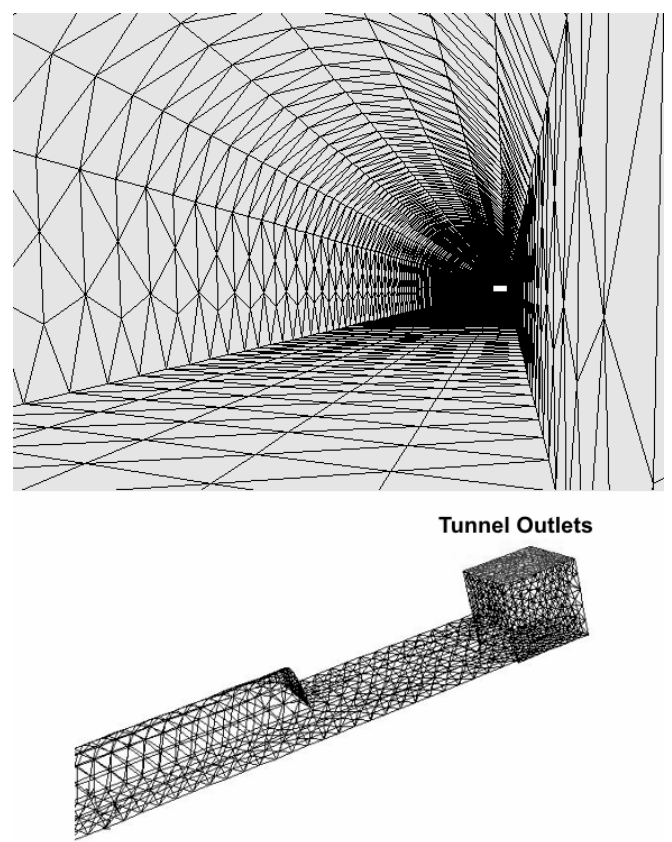

Figure 5. Meshed definition of the tunnel outlets.

The discretization of the Memorial Tunnel geometry was fixed following typical ratios of total number of cells per overall tunnel length (see [11]). The transversal section was discretized using [20x18] cells, with 275 cells along the longitudinal direction of the tunnel, reaching up to approximately 100000 cells for the whole domain. Besides, tetrahedral cells were employed in the definition of the tunnel geometry. Different zones with refined meshes were also introduced where required: over the fire location to enhance the heat transfer process, and in the jet fan inlets and outlets to improve the description of the momentum transfer. However, the overall number of cells is not excessively large since the interest of the work is placed on the development of a designing tool oriented towards the definition of LVS operative plans in case of fire, with moderate computational resources (CPU time). All the significant zones of the numerical model are illustrated in figures 4 to 6 . Figure 4 details the jet fans zone while figure 5 shows the domain outlets. Notice how an additional cubic zone is introduced at both tunnel portals in order to impose horizontal pressure outlet conditions (more neutral than vertical ones). Thus, the effect of gravitational forces modelled in the equations is not compromised with a constant, unrealistic pressure distribution at vertical boundary conditions. Description of the geometry is concluded with the illustration of the grid density in the fire location (figure 6).

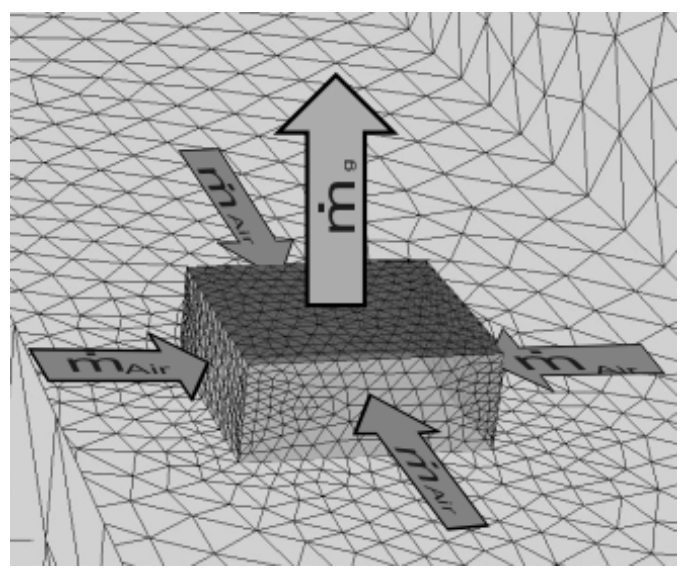

Figure 6. Mesh of the fire site.

Figure 6 also shows the boundary conditions for the heat release rate. Practically, most of these simulations with fire in the literature ([11], [12]) are made without combustion models to avoid the extreme complexity of those models and the uncertainty of the fuel load. The fire is then modelled using a volumetric heat source represented by a heat release rate as a function of the time and by a source mass characterizing the smoke production. Using a rectangular prism, the mass flow rate of the combustion products are introduced in the domain across its upper surface. The injection of mass is balanced with air that is removed across the lateral surfaces of the prism (figure 6). All this mass flow rates are estimated according to the following expressions:

$$
\begin{gathered}
\dot{m}_{g}=\frac{\dot{W}}{C_{p}\left(T_{g}-T_{a m b}\right)} \\
\dot{m}_{\text {air }}=\dot{m}_{g}-\frac{1}{3} \dot{m}_{\mathrm{CO}_{2}}
\end{gathered}
$$

where $\dot{W}$ represents the heat rate, $C_{p}$ is the specific heat of the mixture, $T_{g}$ is the temperature of the combustion gases and $T_{a m b}$ is the surrounding temperature on the fire location. The estimation of the air in (2) is done with estequiometric considerations in the combustion reaction of typical hydrocarbons. Therefore, for a $10 \mathrm{MW}$, the combustion products ( $\left.\dot{m}_{g}\right)$ consisted of a mixture of air ( 0.95 mass fraction) and $\mathrm{CO}_{2}$ (0.05 mass fraction) whereas for a $50 \mathrm{MW}$ fire, the mixture is 0.91 mass fraction of air and 0.09 mass fraction of $\mathrm{CO}_{2}$ (adapted from [4]). This mixture has different temperatures depending on the heat release: for instance, in the case of $10 \mathrm{MW}$, the input temperature is $573.15 \mathrm{~K}$; and for $50 \mathrm{MW}$ this temperature is $950 \mathrm{~K}$ (adapted from [13]). Complementary, radiation has not been modelled, so a reduction of the heat release rate has been introduced instead to take into account radiating features of the heat power. Thus, a reduction of the $35 \%$ of the total heat input is considered as a good estimation for the 
radiation process ([13]). Despite of these constraints, the resulting $\mathrm{CO}_{2}$ and temperature distributions were reasonably correct using this procedure, except in the proximity of the fire location. An additional energetic consideration is the thermal conductivity that must be imposed in the endwalls in order to predict a real temperature distribution inside the tunnel. Considering the wall thickness and the material composition (dolomite), a typical value of $1.75 \mathrm{~W} / \mathrm{mK}$ was fixed to rule the conduction heat transfer from the inner conditions of the wall to the external low temperature of the rock. Additionally, CFD simulations were executed introducing the total heat release from the start of the simulation (10 MW in case of test $606 \mathrm{~A}$ and 50 MW for both $612 \mathrm{~B}$ and 611 cases), following exactly the same operating procedure that was used in the Memorial Tunnel experiments.

The rest boundaries are both pressure and velocity conditions. At the tunnel portals, "free" boundaries were specified in the horizontal top surfaces of those added cubic volumes, imposing a zero gauge pressure to simulate atmospheric conditions outside the tunnel. It has been supposed that no meteorological differences could be found between both tunnel portals. Otherwise, a pressure gradient should be introduced in the operating conditions. For the fan intake, a positive velocity value of $34.2 \mathrm{~m} / \mathrm{s}$ was set to induce the corresponding air suction, whereas at the fan discharge, a negative value was fixed to generate the jet structure outgoing from the fan section.

An unsteady solution was implemented to trace the evolution of the combustion products all over the tunnel geometry. A computational time step was adjusted according to the predominant mechanisms (time and velocity scales), resulting in typical values around 1 second that assure stability criteria for the time-marching numerical solution. The overall time simulation, including both response delay and operating sequence of the fans, reached up to 10 minutes, according to the experimental conditions. The number of iterations has been adjusted to reduce the residuals to below an acceptable value at each time step. In particular the ratio between the sum of the residuals and the sum of the fluxes for a given variable in all the cells is reduced to the value of $10^{-4}$ (four orders of magnitude). The simulation was executed through parallel computations over a 4 PC cluster, 1.8 $\mathrm{GHz}, 512 \mathrm{MB}$ RAM, requiring approximately 50 hours of CPU time to solve the 10 minutes evolution. Every 15 time steps, the three-dimensional flow field was stored, including velocity vectors, pressure distribution, temperature and species concentration (air and $\mathrm{CO}_{2}$ ) or density and turbulent variables, in order to obtain time history graphs in a forward post-processing.

\section{RESULTS}

Results of both CO concentration and temperature distribution are significant because they can give valuable information about the environmental conditions in the tunnel. The $\mathrm{CO}$ concentration is directly obtained from the $\mathrm{CO}_{2}$ results of the model.
Approximately, there is $1 \mathrm{ppm}$ of $\mathrm{CO}$ for every $20 \mathrm{ppm}$ of $\mathrm{CO}_{2}$, in case of non-estequiometric combustions ([4]). Major interest in $\mathrm{CO}$ concentration derives from the potential danger of both substances: while $\mathrm{CO}_{2}$ is basically a contaminant gas, the monoxide is a toxic gas. Thus, large concentrations of dioxide may lead to suffocate, but a relative small proportion of monoxide is lethally poisonous. As a consequence, the simulation makes possible to detect a priori the maximum levels of toxicity and thresholds of human resistance to high temperatures in a fire incident. Then, it can be predicted if irreversible damage will be produced to the people involved in a hypothetical accident. Complementary, this information may determine if the design of the LVS is correct or, if it is not the case, it may be useful to define corrective actions in order to improve the rescue plans.

\section{CO concentration results:}

The severe toxicity of $\mathrm{CO}$ implies that large concentrations of the gas can not be accumulated inside the tunnel for long periods of time. Some authors ([14]) have established that an exposure of 30 minutes to $\mathrm{CO}$ concentration rates of $3000 \mathrm{ppm}$, measured in the upper region of the tunnel, would cause heart and breathe failure, leading to death if the exposure is more prolonged. As a consequence, since major $\mathrm{CO}$ concentrations are always found near the tunnel ceiling, and considering that the real affection zone comprises $6.5 \mathrm{ft}$ height from the road for the people trapped inside, the maximum tolerable rate of $\mathrm{CO}$, which may be used as a criterion for considering smoke-free regions, can be established in 500 ppm ([14]).

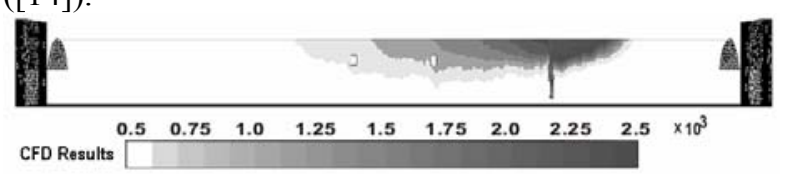

Figure 7.a. Test 606A

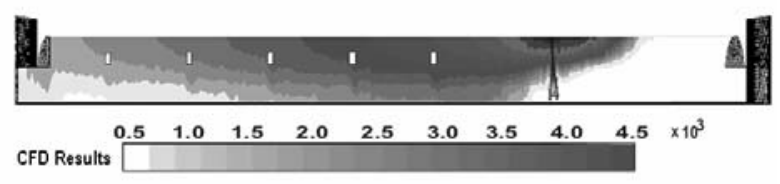

Figure 7.b. Test 612B

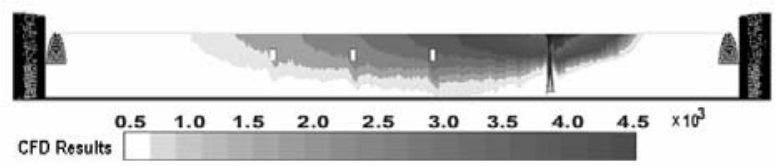

Figure 7.c. Test 611

Figure 7. Longitudinal distribution of $\mathrm{CO}$ concentration (higher than 500 ppm) in the tunnel symmetry plane. Initial conditions for the LVS.

In order to present the behaviour of the smoke, longitudinal contours of $\mathrm{CO}$ concentration along the symmetry plane of the gallery will be shown. Only concentration over $500 \mathrm{ppm}$ is represented with 
colormap, considering that all the zones under that limit are smoke-free spaces (not coloured). In order to appreciate the maps in a better way, the tunnel has been severely distorted in respect to the longitudinal scale.

Figure 7.a shows results in case of a $10 \mathrm{MW}$ fire with a reaction time of 5 minutes (test No. 606A). CO concentration inside the tunnel ranges between 500 and $2500 \mathrm{ppm}$ when 300 seconds have elapsed from the beginning of the fire. Due to the ascending slope in the direction of the north portal, minimal smoke propagation is assured towards the opposite direction. As a consequence, the smoke will tend to propagate towards the north portal, if the thermal input is sufficiently high to generate an important mass of combustion products. Since the heat rate of this test is relatively low, the smoke cannot reach the north portal and a large smoke-free region is established along the tunnel. Therefore, this will be the initial situation that must be managed by the LVS.

Figure 7.b shows $\mathrm{CO}$ concentration in case of 50 MW, 5 minutes after the beginning of the fire incident (test No.612B). Obviously, due to a major heat release, maximum values of concentration are reaching up to $4500 \mathrm{ppm}$. In addition, when compared with the previous figure, the influence of the increase in the power input is clearly illustrated. The $\mathrm{CO}$ fills all the section between the fire location and the North portal, seriously compromising the operative sequence of the forthcoming ventilation start up.

Finally, figure 7.c is included to show the importance of the reaction time of the LVS (test No.611), i.e. the delay time between the beginning of the fire and the starting sequence of the ventilation system. Notice the dramatic difference in the smoke propagation between figures 7.b and 7.c. When only two minutes have passed, no enough amounts of gases have been produced to be propagated to the North portal, facilitating the LVS performance. On the contrary, after 5 minutes, the whole gallery is flooded with toxic gases.

Figure 8 analyzes the effectiveness of the LVS for the three cases modelled in the present work. The initial conditions that the LVS must face up were described in figure 7 . Now, the starting sequence of the fans has been turned on, and the simulation is executed unsteadily until the steady state is reached. The ventilation system operates from North to South portal, inducing an air current that sweeps out the smoke to the nearest portal of the fire location. Thus, the largest part of the tunnel (from the North portal to the fire location) will be assured with fresh-air, reducing the $\mathrm{CO}$ concentration to smaller regions between the fire location and the South portal. The asymptotic state of the smoke propagation when the LVS is switched on is shown in figure 8 . This state corresponds to an overall simulation of 10 minutes after the start of the fire incident. In the 606A test (fig. 8.a) only the two fans, placed far away from the fire were turned on, while tests $612 \mathrm{~B}$ and 611 (fig. 8.b and 8.c) were simulated using five jet fans with different layouts. The two operative jet fans in test 606A are not symmetrically positioned inside the tunnel (this avoids the use of a symmetric domain in the numerical modelling). In the case of test $612 \mathrm{~B}$, the five jet fans of the symmetry plane are working in a row, while for the 611 test, the two closer fans to the fire are operative, supported by the next group of 3 fans. Despite of being started at different instants, all the cases show that the LVS has managed successfully the smoke dispersion inside the tunnel, assuring an optimal smoke-free region from the fire location to the North portal. When the heat input is moderate (10 MW in test 606A), a balance between the production of toxic gases and its transport due to the air current is established, avoiding the presence of high $\mathrm{CO}$ concentration also in the bottom part of the tunnel close to the South portal. The final conditions are quite acceptable, even though this non-symmetrical configuration of the operative fans is clearly inefficient. On the other hand, when the heat release is increased to $50 \mathrm{MW}$ (tests $612 \mathrm{~B}$ and 611), there is a higher smoke production and the drag capacity of the air current is not able to clean off all the bottom zones of the tunnel. Therefore, the heat release has a definitive influence in the propagation velocity of the combustion gases and, as a consequence, in case of high heat rates, the response of the LVS should be small. Also, notice how small differences are appreciable between figures 8.b and 8.c, though similar operating conditions of the LVS and the same heat rate were fixed in both cases. However, different configuration of the operative fans (figure 3) implies different flow patterns inside the tunnel and the final $\mathrm{CO}$ concentration in the symmetry plane varies between the tests.

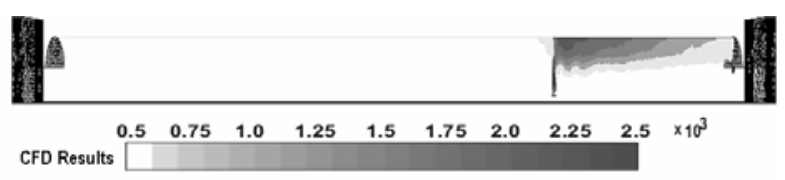

Figure 8.a. Test 606A

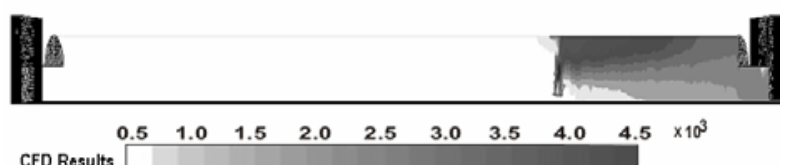

Figure 8.b. Test 612B

繁

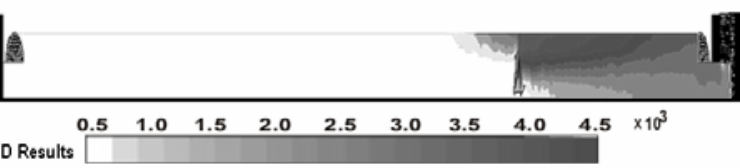

Figure 8.c. Test 611

Figure 8. Longitudinal distribution of $\mathrm{CO}$ concentration (higher than $500 \mathrm{ppm}$ ) in the tunnel symmetry plane. Steady-state conditions for the LVS.

Finally, figure 9 is introduced to illustrate the temporal evolution of the test $612 \mathrm{~B}$, in which the five central jet fans are sweeping out the smoke in a row. When the LVS is started at 300 seconds, the propagation of the smoke towards the North portal is 
suddenly stopped and the contribution of every fan is clearly manifested in the maps. Obviously, those fans located far away from the fire location dissipate the smoke better than those placed beneath the heat release.

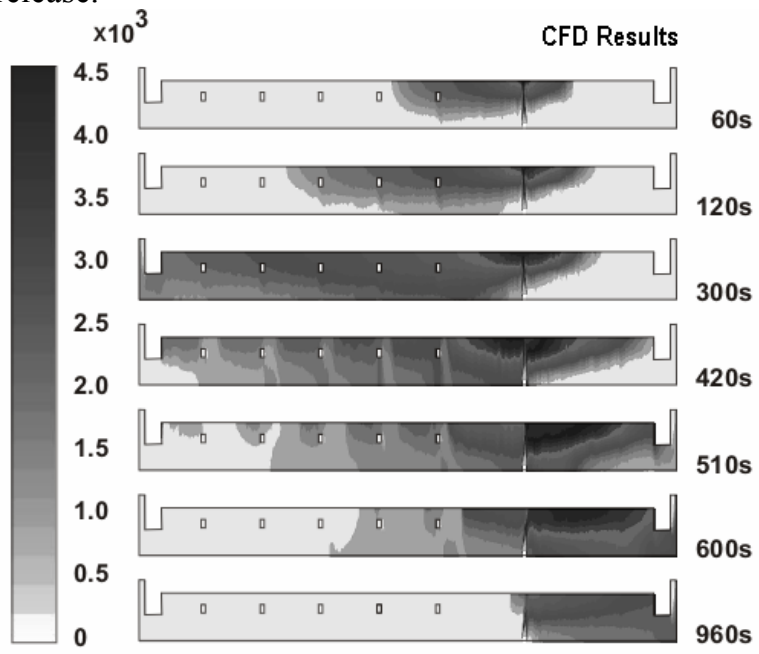

Figure 9. Temporal evolution of CO concentration in the tunnel symmetry plane. Test No. 612B.

\section{Temperature results:}

High temperatures can be also extremely dangerous for the safety of people involved in a fire incident inside a road tunnel. The LVS introduces cold fresh-air that is also contributing to reduce the thermal map inside the gallery. Since the system must keep a bearable temperature in the affection zone of the people, temporal results of the temperature distribution inside the tunnel are extremely useful to observe the goodness of the LVS.
These temporal results will be compared with experimental distributions in order to validate the numerical modelling. Besides, the analysis of the temperature evolution will provide insight in the transport mechanisms of the combustion gases as a function of the LVS parameters.

Test 606A. Figure 10 shows the temperature distribution just before the ventilation system is turned on. Different contour lines of temperature have been represented along the tunnel. The contour line of $140^{\circ} \mathrm{F}$ (approx. $60^{\circ} \mathrm{C}$ ) is considered as the threshold of human tolerance, so it has been boldly marked in the drawings. The contour line of $70^{\circ} \mathrm{F}$ (approx. $21^{\circ} \mathrm{C}$ ) is also interesting, because it begins to separate cold zones from hot regions. The Memorial results exhibit a typical stratification of the smoke region in its first stages. In addition, the $70^{\circ} \mathrm{F}$ contour line is maintained at a constant height of $13 \mathrm{ft}$ from the surrounding area of the fire to the loop 213. On the contrary, results from the numerical model predict a $70^{\circ} \mathrm{F}$ contour line crawling all along the tunnel. Discrepancies in both results derive from the existence of refrigeration devices associated to the instrumentation trees, in order to support the extreme conditions inside the tunnel. Since the numerical model is not including the loops, it is reasonable that differences in the temperature may appear in the bottom zones of the tunnel. Close to the ceiling, there is a better agreement between numerical and experimental results, especially in the longitudinal distance of the contour lines: for instance, the $200^{\circ} \mathrm{F}$ line finishing between the loops 307 and 306, or the $140^{\circ} \mathrm{F}$ line reaching to the loop 208 . In any case, the global distribution of temperature is perfectly reproduced in the simulation.
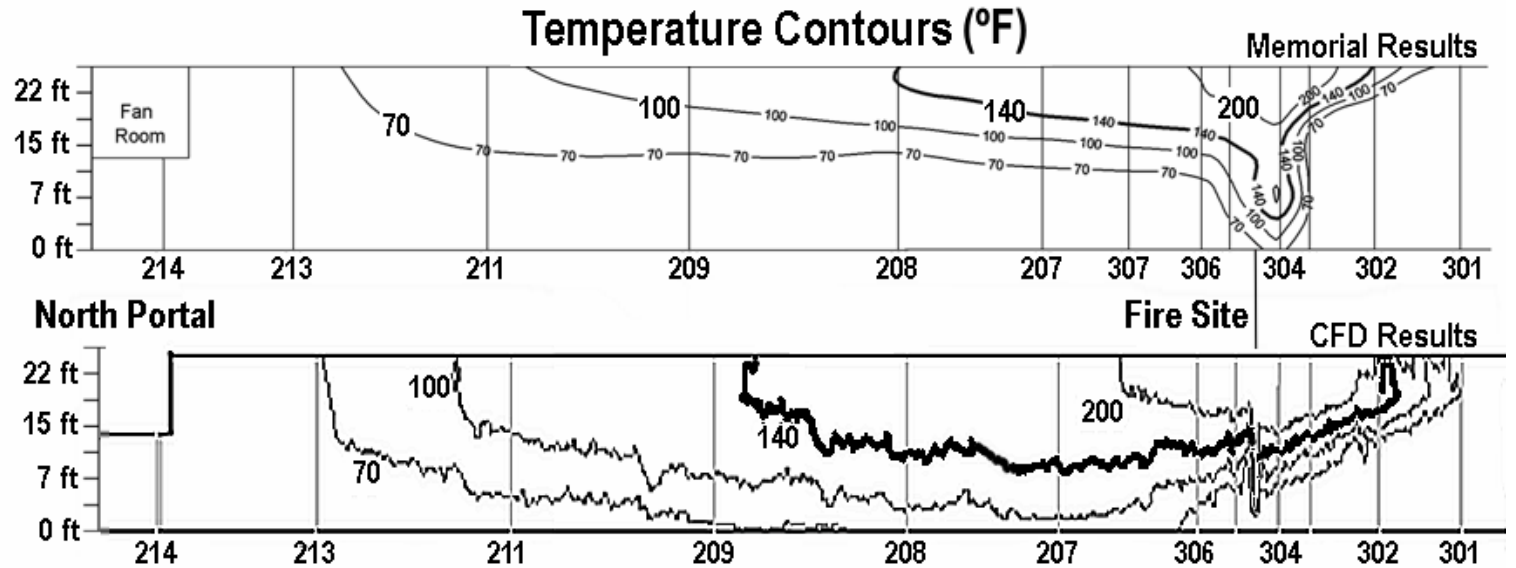

Figure 10. Contours of temperature at $300 \mathrm{~s}$. Experimental-numerical comparison in the case of test 606A.

Figure 11 shows the temperature distribution three minutes after the starting up of the LVS. The air current is sweeping out all the combustion gases towards the South portal, breaking down the smoke stratification. As expected, the numerical model presents a more retarded distribution than that of the experimental results. This effect is a direct consequence of the higher temperatures at the bottom regions of the numerical model, due to the lack of refrigeration of the loops. Therefore, these zones need more time to be cooled than in case of the real conditions, delaying the longitudinal displacement of the smoke. However, the end of the $70^{\circ} \mathrm{F}$ line, placed 
between loops 211 and 209 is well captured in the modelling.

Finally, the steady state distribution of temperature is represented in figure 12 , ten minutes after the beginning of the test. Notice the good agreement between both numerical and experimental distributions, especially from the fire location to the South portal (see the $140^{\circ} \mathrm{F}$ line leaving the domain through the South exit). On the contrary, major differences are placed ahead the fire location, as a clear reminiscence of previous discrepancies in the temporal results (they have been transported against the heat plume of the fire location).

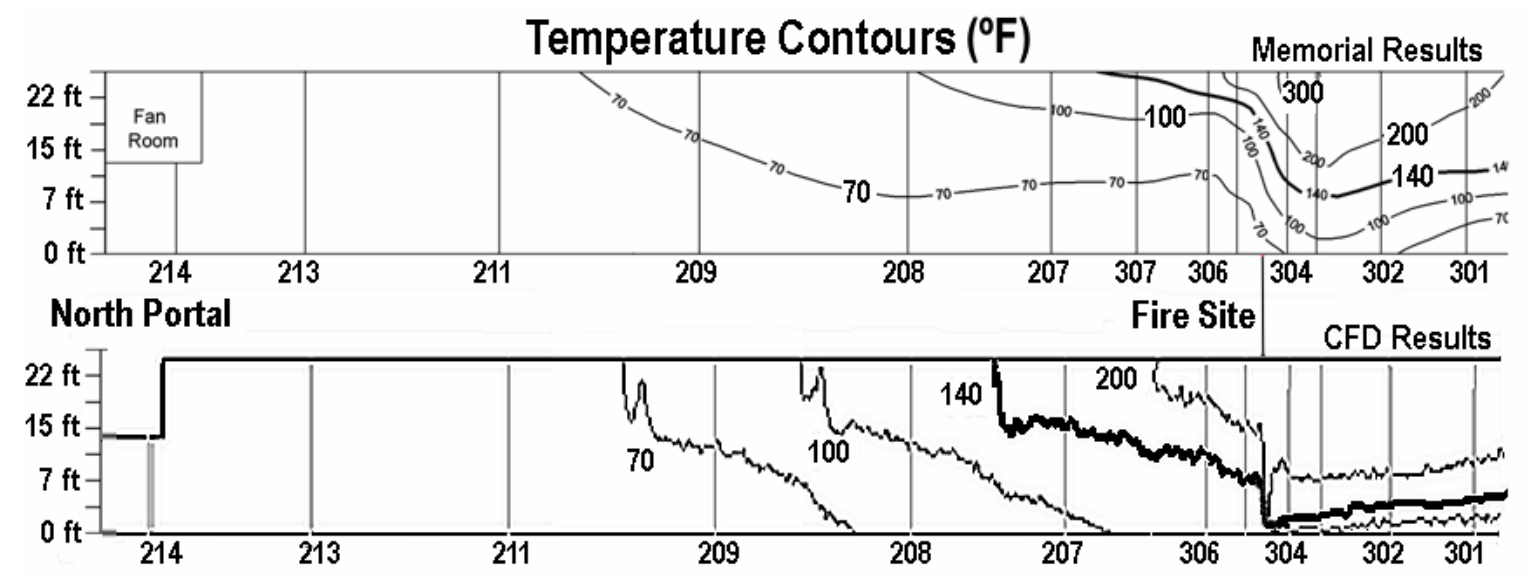

Figure 11. Contours of temperature at $480 \mathrm{~s}$. Experimental-numerical comparison in the case of test 606A.

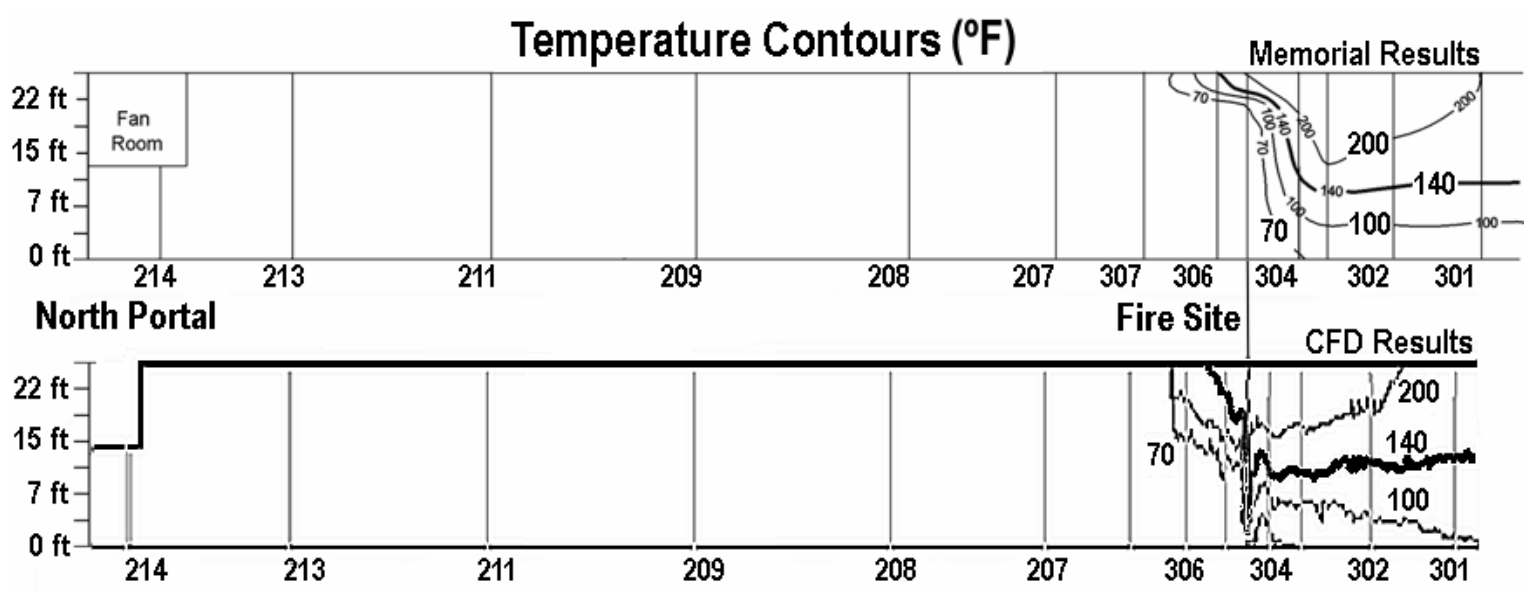

Figure 12. Contours of temperature at $600 \mathrm{~s}$ (steady state). Experimental-numerical comparison in the case of test 606A.

Test 612B. Figure 13 shows the temperature distribution in the case of a $50 \mathrm{MW}$ fire incident. Five minutes after the start, there is a clear smoke stratification, enhanced by the high thermal input of this test. In the experimental results, no line of $70^{\circ} \mathrm{F}$ appears in the maps, indicating the extreme temperature conditions inside the tunnel. Besides, the $140^{\circ} \mathrm{F}$ contour line is placed at 7 - $\mathrm{ft}$ high all over the northern side of the tunnel, seriously compromising the human tolerable threshold. Moreover, it is expected that the height of the $140^{\circ} \mathrm{F}$ contour line should be lower in case of a real fire situation, because no refrigerated loops would be conditioning the thermal map. Thus, in the numerical results, unbearable conditions are extended all over the tunnel (the $140^{\circ} \mathrm{F}$ line is completely stuck on the road), pointing out that a 300 seconds delay is not an acceptable situation for this test. Complementary, the comparison between both contour maps of the figure, reveals significant differences in the maximum temperature obtained at the top of the thermal plume: A $1000^{\circ} \mathrm{F}$ contour line in case of Memorial results and a $600^{\circ} \mathrm{F}$ line in the numerical prediction. Such discrepancies are a direct consequence of the $35 \%$ reduction in the heat input in order to avoid the modelling of the radiation heat transfer in the simulation. Though non-linear, this effect derives also in a notorious reduction of the maximum temperatures inside the domain (in the order of a $30 \%$ ). In any case, the overall thermal distribution is in reasonable agreement with experimental results and the stratification process is perfectly captured by the simulation.

Figure 14 presents the comparison of the temperature results, three minutes after the start of the 
fans operation. Since the five central fans are operating in a row, the gases are completely swept out along the whole tunnel. The thermal tail of the fire is empowered and begins to be extended towards the South portal exit. In addition, the northern side of the tunnel has been perfectly cleaned off gases. Notice the good agreement between the maps on the $70^{\circ} \mathrm{F}$ contour line in the northern side and on the $600^{\circ} \mathrm{F}$ contour line in the southern side.
Finally, figure 15 shows the final state of the smoke concentration when ten minutes have passed since the fire was started. The northern side has been completely cleaned off gases and the thermal plume of the fire is perfectly controlled between loops 306 and 303. Also, the description of the temperature between the fire location and the South portal is analogous in both maps.

\section{Temperature Contours ( $\left.{ }^{\circ} \mathrm{F}\right)$}
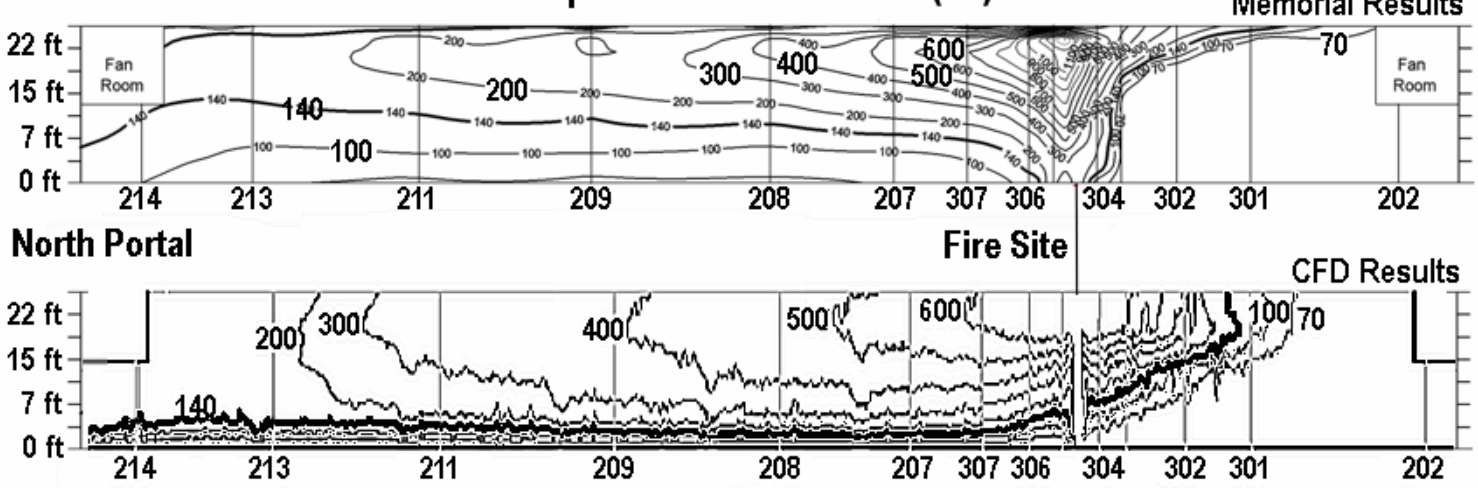

Figure 13. Contours of temperature at 300 s. Experimental-numerical comparison in the case of test $612 \mathrm{~B}$.

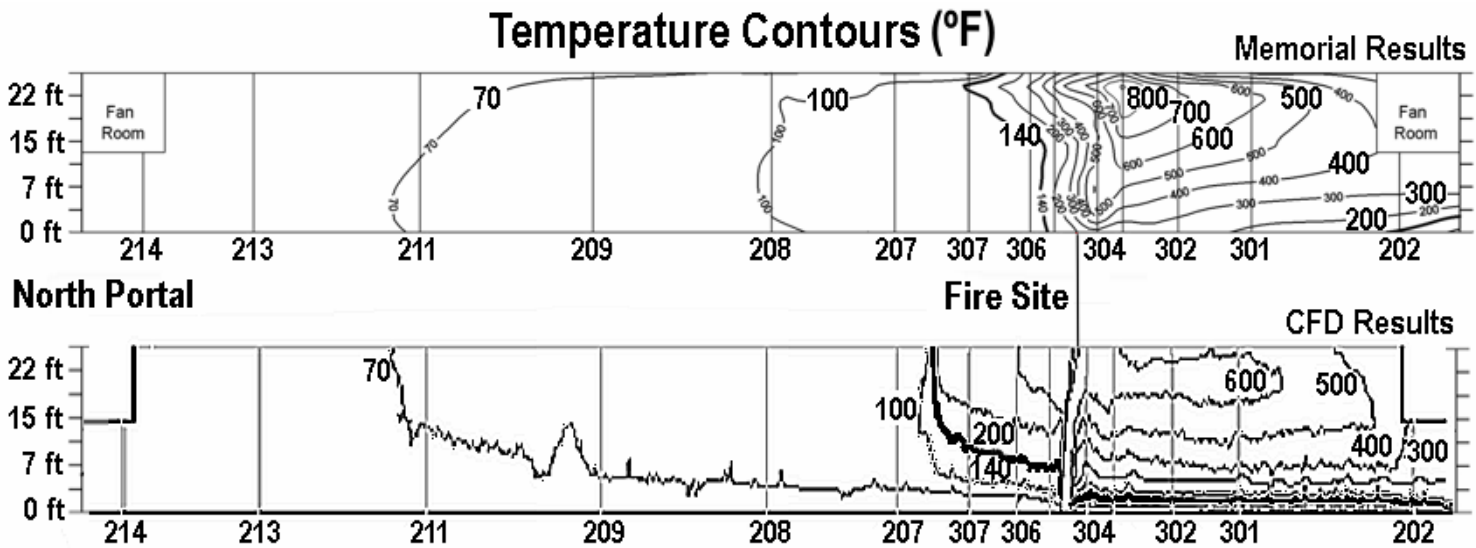

Figure 14. Contours of temperature at 480 s. Experimental-numerical comparison in the case of test $612 \mathrm{~B}$.

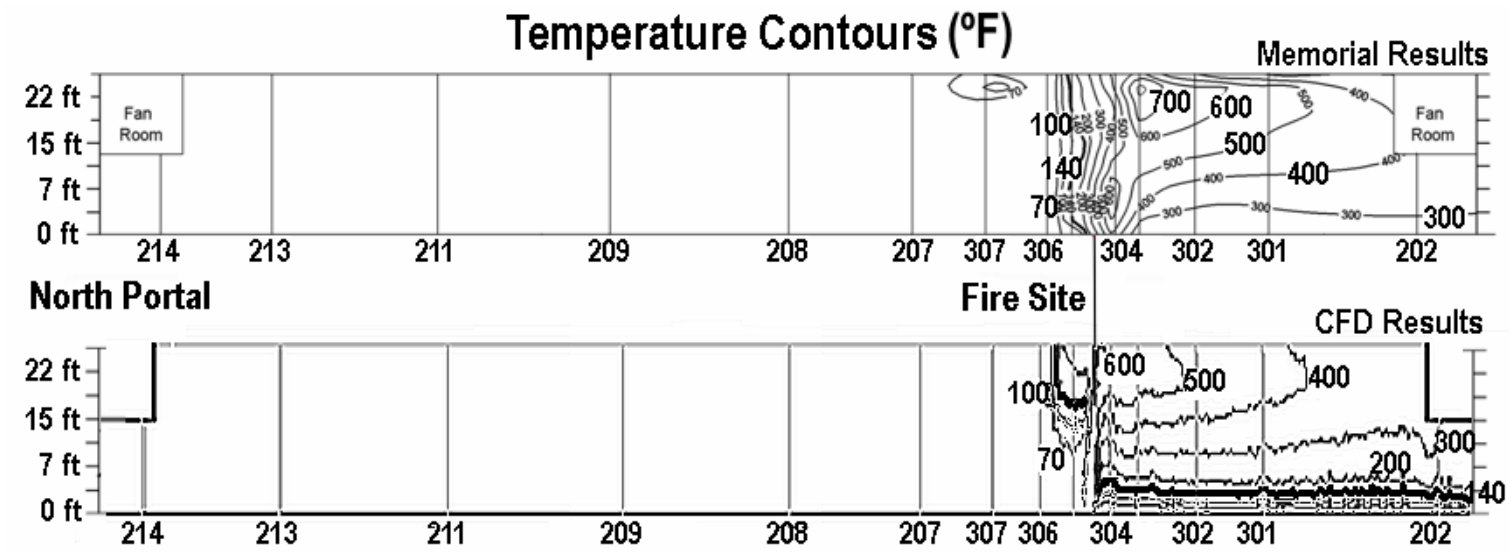

Figure 15. Contours of temperature at 600 s (steady state). Experimental-numerical comparison in the case of test 612B.

Test 611. This section concludes with the presentation of the results in case of a 50MW input fire when the delay time of the LVS has been reduced to 2 minutes. In this case, the smoke propagation is not as severe as 
in the previous test: both experiments and numerical predictions show that the combustion gases have not been extended to the North portal in figure 16. Notice the perfect agreement between the maps when the axial distance of the contour lines are compared. On the other hand, major differences are observable beneath the fire location, due to the restrictive hypothesis employed in the modelling.

Temperature Contours $\left({ }^{\circ} \mathrm{F}\right)$

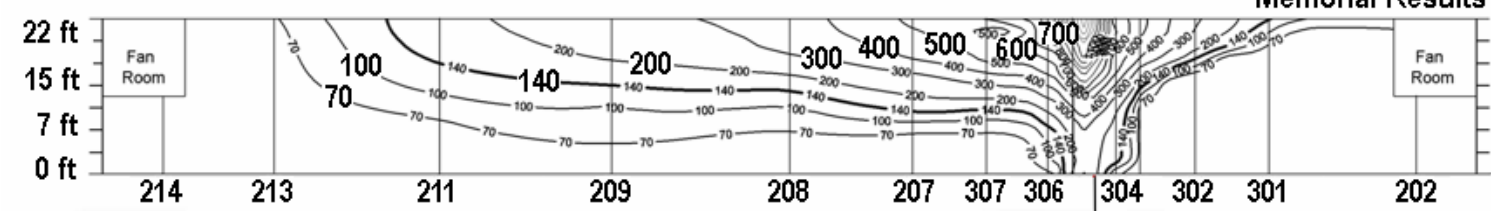

North Portal Fire Site $\quad$ CFD Results

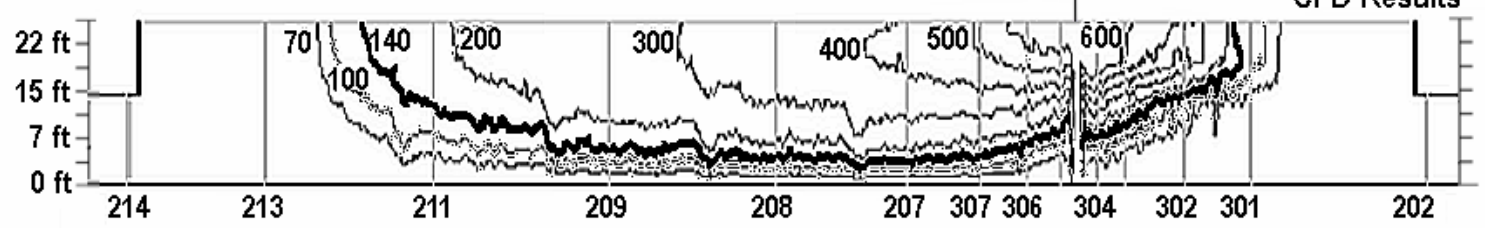

Figure 16. Contours of temperature at 120 s. Experimental-numerical comparison in the case of test 611.

Temperature Contours $\left({ }^{\circ} \mathrm{F}\right)$
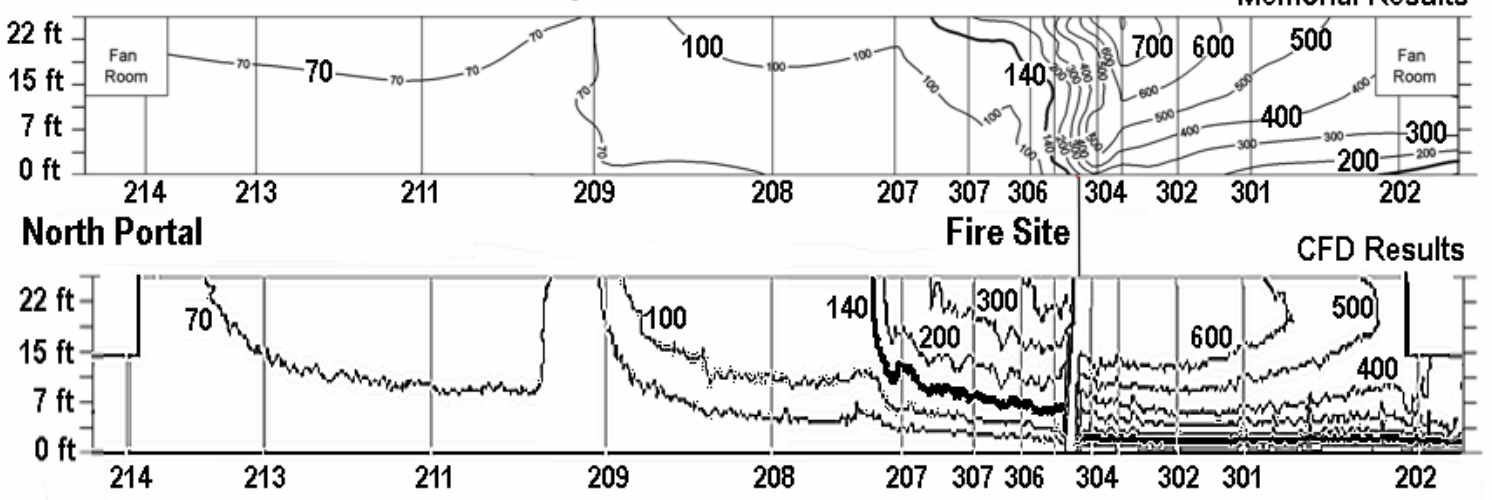

Figure 17. Contours of temperature at 480 s. Experimental-numerical comparison in the case of test 611.

Temperature Contours $\left({ }^{\circ} \mathrm{F}\right)$

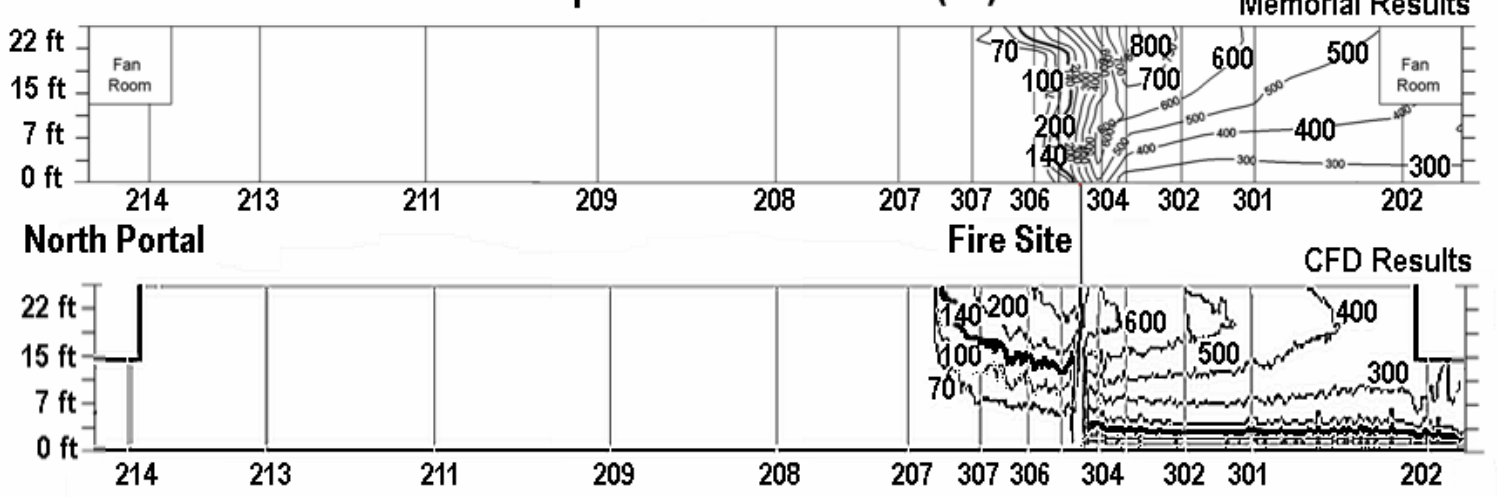

Figure 18. Contours of temperature at $600 \mathrm{~s}$ (steady state). Experimental-numerical comparison in the case of test 611 .

Since the smoke has not flooded the whole gallery when the LVS is turned on, it is no necessary to start all the fans in the longitudinal direction. Then, just two fans close to the fire location and another group of three fans near the loop 209 have been employed to dissipate the smoke inside the tunnel. Figure 17 shows the results six minutes after the fire beginning. Due to the short delay of the LVS, the impact of the refrigeration devices of the loops is not as remarkable when comparing both numerical and experimental results. The thermal budget of the smoke is moderate and hot temperatures are only observed close to the fire 
location. In addition, notice the discontinuity in the $70^{\circ} \mathrm{F}$ contour line in the loop 209 zone, as a direct consequence of the 3-fans group blowing towards the South portal. The smoke pattern is then divided in two regions, creating a recirculation cell between the North portal and the loop 209 that tends to dissipate the combustion gases. This effect has been perfectly predicted by the numerical modelling.

Finally, figure 18 shows the final stationary state for the test 611. Since steady conditions are established, the effect of the refrigerated loops is more significant in the Memorial results. The bottom zones of the tunnel keep a relative low temperature, when compared with the numerical prediction, especially ahead of the fire location. Between the fire location and the South portal, transport mechanisms induced by the air current are predominant and more similar distributions of the contour lines are obtained (i.e., the $300^{\circ} \mathrm{F}$ line, outgoing through the south exit). Anyway, overall agreement is reasonably good.

\section{CONCLUSIONS}

A validation of a numerical model to predict the effectiveness of a longitudinal ventilation system (LVS) has been carried out in the present paper. This model consists in an application of the $3 \mathrm{D}$, unsteady commercial code, FLUENT v6.0, that solves the Navier-Stokes equations in case of multispecies mixture of gases, implementing a thermal balance, which has been customized in order to simulate the evolution of a fire incident inside a road tunnel. Through an exhaustive comparison with experimental results obtained from the Memorial Tunnel Project, the ability of the numerical model to predict the smoke behaviour under free-evolving and ventilated conditions has been tested in different operative situations.

The comparison of temperature results between both experimental and numerical results has shown an overall good agreement, revealing the accuracy and usefulness of CFD modelling in order to predict the effectiveness of LVSs. In particular, longitudinal distributions of temperature were employed for that purpose. Minor differences where found in the vertical profile of the smoke stratification, due to the existence of refrigerated instrumentation in the real tests, whose effects on the thermal map of the tunnel were not implemented in the modelling. However, more significant discrepancies were detected in the maximum temperatures around the fire location, because of the reduction of the heat release in the model, imposed to avoid the modelling of radiating heat transfer. In any case, though these issues should be improved in the future, the present model presents excellent features, especially when the ratio of $\mathrm{CPU}$ time vs. number of tests is considered. Thus, the analysis of the performance of different operating modes for a LVS (i.e., number and layout of working jet fans) can be fulfilled in a short period of time, providing a reasonably accurate prediction of the system goodness.
In addition to this, some basic conclusions can be derived from the analysis of both $\mathrm{CO}$ and temperature distributions. In particular, the fire growth in the initial stages has a significant impact on the environmental conditions that the LVS will have to face off when started. Thus, the reaction time of the system may be a critical parameter if the heat release rate is high enough to compromise the effectiveness of the ventilation system. In any case, since the tunnel is completely cleaned off smoke when steady-state is reached, the LVS has demonstrated to be well-dimensioned with a suitable election of both number and layout of operative jet fans.

In summary, CFD modelling has been presented as a powerful tool to predict the flow behaviour in a road tunnel under fire conditions. Hence, the longitudinal ventilation system has been tested and properly modelled, especially when buoyant driven airflows are well-established.

\section{NOMENCLATURE}

$\begin{array}{lll}\text { CFD } & \text { Computational Fluid Dynamics } \\ \mathrm{CO} & \text { Carbon Monoxide } & \\ \mathrm{CO}_{2} & \text { Carbon Dioxide } & \\ \mathrm{LVS} & \text { Longitudinal Ventilation System } & \\ \text { MTFVTP Memorial Tunnel Fire Ventilation } & \text { Test } \\ & \text { Program } & \end{array}$

$\alpha_{i} \quad$ Local mass fraction for specie $\mathrm{i}$

$C_{p} \quad$ Mixture specific heat $\left[\mathrm{m}^{2} / \mathrm{s}^{2} \cdot \mathrm{K}\right]$

$\dot{m}_{\text {air }} \quad$ Air mass flow rate $\left[\mathrm{m}^{3} / \mathrm{s}\right]$

$\dot{m}_{\mathrm{CO}_{2}} \quad$ Carbon Dioxide mass flow rate $\left[\mathrm{m}^{3} / \mathrm{s}\right]$

$\dot{m}_{\text {gas }} \quad$ Gas mass flow rate $\left[\mathrm{m}^{3} / \mathrm{s}\right]$

$N \quad$ Total number of fluid phase chemical species

$N_{t h} \quad$ Nth-number of fluid phase chemical specie

$T_{a m b} \quad$ Surrounding temperature on the fire location [K]

$T_{g} \quad$ Temperature of the combustion gases [K]

$\dot{W} \quad$ Heat rate [W]

\section{REFERENCES}

[1] Vantelon, J.P., Guelzim, A., Quach, D., Son, D.K., Gabay, D. and Dallest, D., 1991. "Investigation of fire-induced smoke movement in tunnels and stations: an application to the Paris metro". 3th International Symposium on Fire Science, IAFSS.

[2] Oka, Y. and Atkinson, G.T., 1995. "Control of smoke flow in tunnel fires". Fire Safety journal, 25, pp.305-322, 1995.

[3] Saito, N., Yamada, T., Sekizawa, A., Yanai, E., Watanabe, Y. and Myazaki, S., 1995. "Experimental study on fire in a wind tunnel with a reduced scale model". 2nd International Conference on Safety in Road and Rail Tunnels, ITC, Granada. 
[4] EUREKA-Project EU 499: Firetun, 1995. Fires in Transport Tunnels: Report on Full-Scale Tests. Studiengesellschaft Stahlanwendung e.V., Düsseldorf.

[5] Bechtel / Parsons Brinckerhoff, 1995. "Memorial Tunnel Fire Ventilation Test Program, Comprehensive Test Report”. Massachusetts Highway Department.

[6] Chow, W.K., 1998. "On smoke control for tunnels by longitudinal ventilation". Tunnelling and Underground Space Technology, Vol.13 (3), 1998.

[7] Jojo, S.M., Chow, W.K., 2003. "Numerical studies on performance evaluation of tunnel safety systems". Tunnelling and Underground Space Technology, Vol.18, 2003.

[8] Gao, P.Z., Liu, S.L., Chow, W.K., Fong, N.K., 2004. "Large eddy simulations for studying tunnel smoke ventilation". Tunnelling and Underground Space Technology, Vol.19, 2004.

[9] Hu, L.H., Huo, R., Peng, W., Chow, W.K., Yang, R.X., 2005. "On the maximum smoke temperature under the ceiling in tunnel fires". Tunnelling and Underground Space Technology, Vol.21, 2006.

[10] Carvel, R.O., Beard, A.N., Jowitt, P.W., 2001. "The influence of longitudinal ventilation systems on fires in tunnels". Tunnelling and Underground Space Technology, Vol.16, 2001.

[11] Ballesteros, R., Santolaria, C., Blanco, E., 2006. "Influence of the slope in the ventilation semitransversal system of an urban tunnel". Tunnelling and Underground Space Technology, Vol.21, 2006.

[12] Modic, J., 2003. "Fire simulation in road tunnels". Tunnelling and Underground Space Technology, Vol.18, 2003.

[13] PIARC, Committee on Road Tunnels, 1999. "Fire and Smoke Control in Road Tunnels".

[14] Purser, D., 1993. "Modelling Toxic and Physical Hazard in Fire". Interflam. 
This document is a pre-print version of the scientific paper published by Elsevier. It has been released by the authors to fulfill all the publisher requirements established for Article Sharing: https://www.elsevier.com/about/policies/sharing

\section{(9) $\Theta \Theta \Theta$}

(C) 2019. This manuscript version is made available under the Creative Commons Attribution-NonCommercial-NoDerivatives 4.0 International License (CC-BY-NC-ND 4.0 license) http://creativecommons.org/licenses/by-nc-nd/4.0/ 